\title{
The Use of Uroflowmetry to Diagnose Recurrent Stricture After Urethral Reconstructive Surgery
}

\author{
Bradley A. Erickson, Benjamin N. Breyer, and Jack W. McAninch* \\ Department of Urology, University of California-San Francisco, San Francisco, California
}

\begin{abstract}
Purpose-The ability of uroflowmetry to diagnose recurrent stricture disease after urethroplasty has not been fully investigated.

Materials and Methods-Our routine post-urethroplasty monitoring includes retrograde urethrogram and voiding cystourethrogram at 3 and 12 months, in addition to uroflowmetry at 3month intervals for a year. All uroflowmetry data, including maximum flow rate, voided volume and voiding curve shape, as well as retrograde urethrogram/voiding cystourethrogram and voiding symptom data are stored in a prospectively maintained urethroplasty database that was analyzed for patients with postoperative retrograde urethrogram/voiding cystourethrogram and satisfactory uroflowmetry in the same period. Uroflowmetry data points and urinary symptoms were compared with corresponding findings on retrograde urethrogram/voiding cystourethrogram to determine the ability of uroflowmetry to predict recurrence.
\end{abstract}

Results-A total of 278 men (68\%) met study inclusion criteria, of whom 63 (23\%) had recurrent stricture. Using a maximum flow rate of less than $10 \mathrm{ml}$ per second resulted in only $54 \%$ test sensitivity to predict recurrence. The highest sensitivity and negative predictive value (each 99\%) were achieved when all men with symptoms and/or obstructed flow curves were evaluated. Symptoms alone had a high specificity $(87 \%)$, sensitivity $(88 \%)$ and negative predictive value $(95 \%)$.

Conclusions-Uroflowmetry is an adequate test to screen for postoperative stricture recurrence but only when the voiding curve and urinary symptoms are also evaluated. The flow rate alone does not appear to be a reliable tool to evaluate stricture recurrence.

\section{Keywords}

urethra; urethral stricture; urodynamics; recurrence; reconstructive surgical procedures

Urethroplasty is the gold standard to manage urethral stricture disease with greater than $90 \%$ success for most repair types. Urethral stricture is generally diagnosed when combined RUG and VCUG is done in a patient with obstructive urinary symptoms and suspicion of urethral stricture. ${ }^{1,2}$ This combination of radiological tests allows visualization of the entire urethra and, thus, the entire extent of the urethral stricture.

Combined RUG/VCUG is also an excellent method to diagnose recurrent urethral stricture disease after urethroplasty. However, given the high success rates of urethral reconstructive surgery, the need for all men to undergo postoperative radiological testing has been called into question. ${ }^{3}$ Since RUG/VCUG is costly and some-times logistically difficult to perform,

\footnotetext{
(C) 2010 by American Urological Association Education and Research, Inc.

* Correspondence: 1001 Potrero Ave., 3A20, San Francisco, California 94110 (telephone: 415-206-4030; FAX: 415-206-5153; jmcaninch@urology.ucsf.edu)..
} 
and exposes men to radiation, many reconstructive urologists have begun to use less invasive postoperative monitoring protocols, such as periodic UF, or they simply follow patient voiding symptoms for evidence of recurrence. . $^{3,4}$ To our knowledge these protocols have never been tested against the radiological test findings.

Our routine postoperative monitoring protocol after urethral reconstructive surgery includes RUG/VCUG with UF at the same visit at 3 and 12 months. We compared the gold standard RUG/VCUG to UF and assessed the ability of UF alone to diagnose recurrent stricture. The study hypothesis was that patient UF findings would likely correlate closely enough with RUG/VCUG findings, such that UF would be appropriate as a screening test for recurrent stricture.

\section{MATERIALS AND METHODS}

\section{Patient Selection}

We analyzed our prospectively collected, institutional review board approved urethroplasty database to identify all men who underwent urethral reconstructive surgery from 1999 to 2008. Of this initial group men who underwent postoperative RUG/VCUG and UF within 1 week of each other were included in the study. If men had multiple RUG/VCUG/UF sets that met study inclusion criteria, only the most recent set was included. Also, only UFs with VV greater than $150 \mathrm{ml}$ were included in analysis since this is the minimal amount of voided urine needed for a reliable test. ${ }^{5}$ Men without sufficient UF and VCUG/RUG available were excluded from study.

\section{Radiological Testing}

We performed all postoperative radiological testing in an office based fluoroscopy suite. RUG is done first with a catheter tipped syringe and the patient slightly lateral. At this examination we attempt to distend the entire anterior urethra to the external sphincter level. We catheterize the urethra using an $8 \mathrm{Fr}$ latex catheter. The bladder is filled with contrast medium until the patient feels the urge to void. The catheter is then removed and VCUG is done. At this examination we give particular attention to the posterior urethra, which generally distends during voiding, and to any areas that may have appeared abnormal on retrograde study. If any concerning urethral narrowing is seen during either study, especially in the area where urethral reconstruction was performed, it is usually investigated by cystoscopy and considered recurrence when narrowing is confirmed during endoscopic evaluation.

\section{Uroflowmetry}

All men are asked to abstain from urination before the visit, if possible, so that the most accurate UF values from larger volume voids can be obtained. Also, at the visit all patients are asked about any new voiding difficulties, ie interval slowing of flow, hesitancy, frequency or intermittency, which was recorded in the database. We used a UFS-2005 Flowstar uroflowmeter (Medical Measurement Systems, Dover, New Hampshire) for all UF testing. UF data that are recorded prospectively include the maximum flow rate in $\mathrm{ml}$ per second, voiding curve shape and VV in ml. Patients who do not void greater than $150 \mathrm{cc}$ are asked to repeat the test after consuming water. If any patient is unable to void greater than $150 \mathrm{cc}$, bladder ultrasound is generally done in the office to ensure that the patient is not in urinary retention. Voiding curves were recorded as bell-shaped (normal) or flat (obstructed) and read by a single urologist (JWM) who was not blinded to RUG/VCUG results or patient voiding symptoms. 


\section{Statistical Analysis}

RUG/VCUG findings in each patient were compared to the corresponding UF study to determine which data points or combinations of data points on UF best predicted recurrence. We calculated sensitivity, specificity, PPV and NPV. We also assessed subjective patient urinary symptoms for the ability to predict recurrence. Stata ${ }^{\circledR} 11$ was used for statistical analysis. We used the paired and unpaired t test, and chi-square analysis as appropriate with statistical significance considered at $\mathrm{p}<0.05$.

\section{RESULTS \\ Demographics}

A total of 407 men underwent urethral reconstruction in the study period, of whom 278 (68\%) met study inclusion criteria. Men were excluded from analysis due to absent postoperative RUG/VCUG and UF done during the same period (74) or due to UF without VV greater than $150 \mathrm{ml}$ and/or an un-interpretable voiding curve (55). Table 1 lists demographics in the 129 and 278 men excluded from and included in the study, in whom mean \pm SD age was $44.9 \pm 17.1$ and $42.5 \pm 15.9$ years $(\mathrm{p}=0.18)$, and median stricture length was 2.3 (range 0.5 to 19 ) and 2.5 (range 0.5 to 22$) \mathrm{cm}(\mathrm{p}=0.64)$, respectively. Excluded men were less likely to have recurrent stricture $(\mathrm{p}<0.0001)$ and they had shorter followup than included men $(20 \pm 22.7$ vs $13.2 \pm 22.7$ months, $p=0.0063)$. These findings are explained by our observation that men without postoperative urinary problems tend to be less compliant with our followup protocols.

\section{Postoperative Recurrent Stricture}

We identified recurrent stricture postoperatively in 63 men (23\%). Those with recurrence had longer strictures (0.0001), and were more likely to undergo patch graft procedures $(\mathrm{p}=$ 0.007 ) and less likely to undergo anastomotic procedures ( $\mathrm{p}$ \.0001) than those without recurrence (table 2).

\section{Uroflowmetry}

Men with recurrent strictures had a lower postoperative maximum flow rate $(p=0.0001)$ and VV ( $\mathrm{p}=0.017)$, and were more likely have an obstructive voiding curve and voiding symptoms (each $<0.0001)$ than men without recurrence. Followup was significantly longer in men with recurrent stricture $(\mathrm{p}=0.0001$, table 2$)$.

\section{UF vs RUG/VCUG Findings}

Table 3 lists sensitivity, specificity, PPV and NPV calculations for all test parameters. The highest test sensitivity (99\%) to predict recurrent stricture was calculated when all men with urinary symptoms and/or an obstructive voiding curve were evaluated. This means that if we had done RUG/VCUG only in men with voiding symptoms or with a UF study that revealed an obstructive voiding curve, we would have missed recurrent stricture in only $1 \%$. This combination of testing had the highest NPV (99\%), meaning that if a patient lacked voiding symptoms and had a nonobstructive/bell-shaped voiding curve, he had only a $1 \%$ chance of recurrent stricture.

Had we used the most stringent criteria for RUG/VCUG, including maximum flow less than $10 \mathrm{ml}$, an obstructive voiding curve and urinary symptoms, this would have resulted in only $51 \%$ sensitivity, which would mean that almost half of the recurrences would have been missed. However, this combination of tests resulted in the highest test specificity (98\%) and positive predictive value $(90 \%)$, meaning that if a man had all of the mentioned criteria, he would have a $90 \%$ chance of recurrent stricture being found on VCUG/RUG. 
The sensitivity and specificity of evaluating symptoms alone was $87 \%$ and $88 \%$, respectively. In our study population only 9 men (14\%) with recurrent stricture were asymptomatic, resulting in a high NPV (95\%). Conversely 25 men (12\%) without recurrence noticed voiding symptoms postoperatively, resulting in a low PPV (71\%).

\section{DISCUSSION}

Radiographic testing after urethroplasty provides excellent detail of the reconstructed urethra. ${ }^{6}$ It can also provide peace of mind for the surgeon and the patient since it vividly shows a widely patent urethra after successful repair. However, in the era of cost conscious, minimally invasive medicine is routine RUG/VCUG really necessary? With a success rate of greater than $90 \%$ most of these men proceed to unnecessary testing that exposes them to radiation as well as to other complications, such as urinary tract infection.

To answer that question we looked at urethroplasties done at our institution in a 10-year period. We compared findings from our routine 3 and 12-month postoperative RUG/VCUG studies to those of the concomitant UF and evaluated whether noninvasive UF could supplant radiographic testing. Findings show that UF alone is not a sufficient study to screen for recurrence, especially when we used the maximum flow rate. While specificity of the maximum flow rate improved as the rate was decreased to $10 \mathrm{ml}$ per second, this was at the expense of unacceptably low sensitivity (54\%), which is undesirable for any screening test. Using voiding curve data was more helpful with $93 \%$ sensitivity and $84 \%$ specificity. However, interpretation of the voiding curve is admittedly subjective, is usually done by a nonblinded investigator and would be difficult to recommend as a stand-alone screening tool.

UF has been used to evaluate urinary obstruction in other urological disease processes, most commonly in men suspected of having benign prostatic hyperplasia. In this group a flow rate of less than $10 \mathrm{ml}$ per second has greater than $90 \%$ specificity and PPV to diagnose obstruction. However, to achieve such a high PPV, which is desirable for benign prostatic hyperplasia since it provides information on who could potentially benefit from surgical intervention, sensitivity is again low with most groups reporting only $40 \% .^{5}$

Pediatric urologists have also popularized UF in children after hypospadias repair. In this population a noninvasive way to test for obstruction is ideal since it is generally difficult to perform any manipulative procedure in children without anesthesia. What small studies have shown is that children with a flow rate of $2 \mathrm{SD}$ below the mean or those with a flat voiding curve have a high likelihood of post-repair urethral stricture. ${ }^{7,8}$ Children suspected of having postoperative pathology by UF parameters can then undergo urethral examination with a high likelihood of finding recurrence and of successfully managing recurrent obstruction at the same operative/anesthetic setting.

The adult urethroplasty population is a more complex group to analyze noninvasively. Although multiple studies show significant improvements in UF after successful urethroplasty, they generally mention only mean improvements in the flow rate in the entire group and not individual improvement. ${ }^{4,9}$ A problem with UF in adults is the wide variability among older patients, which is particularly problematic in the post-urethroplasty population. ${ }^{10}$ Since many of these men have had long-standing urethral obstruction, the bladder is often relatively decompensated and the flow rate can be relatively low even without obstruction. ${ }^{11}$ For example, in our series almost $16 \%$ of men without recurrence had a maximum flow rate of less than $15 \mathrm{ml}$ per second, which has been used in some studies as a threshold for urethral imaging. ${ }^{4,9}$ Conversely almost $30 \%$ of men with recurrence had a flow rate of greater than $15 \mathrm{ml}$ per second. Also, since the median age of repair in this study 
was 42 years (range 19 to 85), a large percent of the men had a component of benign prostatic hyperplasia, which further complicates UF data interpretation. These confounding variables make setting a maximum flow rate cutoff at which all men would be evaluated for recurrent recurrence problematic when using UF as a screening measure.

Instead, the most effective way to use UF may be to perform UF preoperatively in all men and then post-urethroplasty UF at 3 months after verifying a patent urethra on RUG/VCUG done the same day. These UF values would effectively represent the patient baseline UF. At subsequent followup visits UF could then be repeated and any deviation from baseline in maximum flow or voiding curve shape would represent possible recurrence and could be evaluated by RUG/VCUG.

Also, monitoring patient voiding symptoms should be an integral part of any followup protocol screening for recurrence. Of study patients $85 \%$ were symptomatic when recurrence was found and only $12 \%$ had symptoms without recurrence. Although we did not and do not routinely use validated questionnaires to monitor symptoms, the American Urological Association symptom index questionnaire was previously studied for use in the urethral stricture population and showed some promise in its ability to monitor urethral status after changes in symptom scores. ${ }^{4,12}$ Adding a questionnaire that generates a voiding score would make monitoring symptoms more objective. Similar to our proposed UF method one could obtain preoperative and postoperative symptom scores, and perform radiographic testing if the score significantly worsens. A combined UF/symptom score protocol was previously evaluated in men who underwent prior direct visual internal urethrotomy with $93 \%$ sensitivity to predict recurrence vs urethral calibration alone. It is likely that if a similar protocol were evaluated prospectively in patients after urethral reconstruction, similar sensitivity would be achieved.

Our study has limitations. Although we used prospectively recorded data, hypothesis generation was retrospective and, thus, any conclusions generated by the study must be evaluated in a dedicated prospective study. Also, 55 men (14\%) were excluded from analysis due to inadequate UF. This happened partly because we did not look at UF prospectively but it may also highlight some inherent difficulties that a urologist may often encounter when trying to use UF in clinical practice. It is often difficult in a busy clinic to obtain accurate readings in all patients, which must be considered when attempting to use UF effectively to predict recurrence. Still, our study findings are important, in that they show that UF alone is not likely sufficient to find all recurrences. However, when combined with symptom evaluation, it may decrease the number of RUG/VCUG studies done when looking for recurrence. Further studies are needed to evaluate the safety and costeffectiveness of such an approach.

\section{CONCLUSIONS}

The ideal followup protocol after urethral reconstructive surgery has not yet been established. While RUG/VCUG is almost 100\% sensitive to diagnose obstruction and recurrence, performing these tests multiple times is probably unnecessary in all patients. Since most men with recurrence in our study presented with voiding symptoms or abnormal UF, using a combination of these tests postoperatively may be an appropriate method to screen for those who need additional radiographic testing. However, UF alone does not appear to be a reliable indicator of recurrent stricture. 


\section{Abbreviations and Acronyms}

$\begin{array}{ll}\text { NPV } & \text { negative predictive value } \\ \text { PPV } & \text { positive predictive value } \\ \text { RUG } & \text { retrograde urethrogram } \\ \text { UF } & \text { uroflowmetry } \\ \text { VCUG } & \text { voiding cystourethrogram } \\ \text { VV } & \text { voided volume }\end{array}$

\section{REFERENCES}

1. Asklin B, Nilsson A, Pettersson S. Functional evaluation of anterior urethral strictures with combined antegrade and retrograde urethrography. Scand J Urol Nephrol. 1984; 18:1. [PubMed: 6719047]

2. Pavlica P, Barozzi L, Menchi I. Imaging of male urethra. Eur Radiol. 2003; 13:1583. [PubMed: 12835971]

3. Meeks JJ, Erickson BA, Granieri MA, et al. Stricture recurrence after urethroplasty: a systematic review. J Urol. 2009; 182:1266. [PubMed: 19683309]

4. Heyns CF, Marais DC. Prospective evaluation of the American Urological Association symptom index and peak urinary flow rate for the followup of men with known urethral stricture disease. $\mathrm{J}$ Urol. 2002; 168:2051. [PubMed: 12394706]

5. Belal M, Abrams P. Noninvasive methods of diagnosing bladder outlet obstruction in men. Part 2: noninvasive urodynamics and combination of measures. J Urol. 2006; 176:29. [PubMed: 16753360]

6. Choudhary S, Singh P, Sundar E, et al. A comparison of sonourethrography and retrograde urethrography in evaluation of anterior urethral strictures. Clin Radiol. 2004; 59:736. [PubMed: 15262549]

7. Garibay JT, Reid C, Gonzalez R. Functional evaluation of the results of hypospadias surgery with uroflowmetry. J Urol. 1995; 154:835. [PubMed: 7609192]

8. Kaya C, Kucuk E, Ilktac A, et al. Value of urinary flow patterns in the follow-up of children who underwent Snodgrass operation. Urol Int. 2007; 78:245. [PubMed: 17406135]

9. Barbagli G, Guazzoni G, Lazzeri M. One-stage bulbar urethroplasty: retrospective analysis of the results in 375 patients. Eur Urol. 2008; 53:828. [PubMed: 18243497]

10. Feneley MR, Dunsmuir WD, Pearce J, et al. Re-producibility of uroflow measurement: experience during a double-blind, placebo-controlled study of doxazosin in benign prostatic hyperplasia. Urology. 1996; 47:658. [PubMed: 8650862]

11. Belal M, Abrams P. Noninvasive methods of diagnosing bladder outlet obstruction in men. Part 1: nonurodynamic approach. J Urol. 2006; 176:22. [PubMed: 16753359]

12. Morey AF, McAninch JW, Duckett CP, et al. American Urological Association symptom index in the assessment of urethroplasty outcomes. J Urol. 1998; 159:1192. [PubMed: 9507830] 
Table 1

Demographics in men included in vs excluded from study

\begin{tabular}{lccc}
\hline & No. Included (\%) & No. Excluded (\%) & p Value \\
\hline Repair: & & & \\
Anastomosis & $142(51)$ & $65(49)$ & 0.80 \\
Flap & $29(10)$ & $18(14)$ & 0.31 \\
Graft & $85(30)$ & $41(32)$ & 0.83 \\
Flap/graft & 11 & 2 & 0.05 \\
Anastomosis/flap & 3 & 3 & 0.34 \\
Anastomosis/graft & 8 & 0 & - \\
Site: & & & \\
Bulbar & $173(62)$ & $82(64)$ & 0.80 \\
Penile & $21(8)$ & $15(12)$ & 0.16 \\
Penile/bulbar & $38(14)$ & $11(8)$ & 0.13 \\
Prostatomembranous & $46(17)$ & $21(16)$ & 0.93 \\
Recurrent stricture & 63 & 10 & $<0.0001$ \\
\hline
\end{tabular}


Table 2

Demographics and overall UF in men with vs without recurrence

\begin{tabular}{|c|c|c|c|c|c|}
\hline & \multicolumn{2}{|c|}{ No Recurrence } & \multicolumn{2}{|c|}{ Recurrence } & p Value \\
\hline No. pts & \multicolumn{2}{|l|}{215} & \multicolumn{2}{|l|}{63} & \\
\hline Mean \pm SD age & \multicolumn{2}{|c|}{$42.1 \pm 16$} & \multicolumn{2}{|c|}{$44.5 \pm 15.5$} & 0.26 \\
\hline $\begin{array}{l}\text { Median cm stricture length } \\
\text { (range) }\end{array}$ & \multicolumn{2}{|c|}{$2.29(0.5-19)$} & \multicolumn{2}{|c|}{$3.5(1-22)$} & 0.0001 \\
\hline \multicolumn{6}{|l|}{ No. repair (\%): } \\
\hline Anastomosis & 123 & (57) & 19 & $(30)$ & $<0.0001$ \\
\hline Flap & 23 & (11) & 6 & $(9.6)$ & 0.79 \\
\hline Graft & 57 & (27) & 28 & $(44)$ & 0.007 \\
\hline Flap/graft & 4 & (2) & 7 & (11) & 0.001 \\
\hline Anastomosis/flap & 2 & $(0.9)$ & 1 & (1.6) & 0.66 \\
\hline Anastomosis/graft & 6 & $(2.8)$ & 2 & $(3.2)$ & 0.87 \\
\hline \multicolumn{6}{|l|}{ No. site (\%): } \\
\hline Bulbar & 142 & $(66)$ & 31 & $(49)$ & 0.019 \\
\hline Penile & 18 & $(8.4)$ & 3 & $(4.8)$ & 0.34 \\
\hline Penile/bulbar & 17 & (7.9) & 21 & (33.3) & $<0.0001$ \\
\hline Prostatomembranous & 38 & (17.7) & 8 & (13) & 0.35 \\
\hline $\begin{array}{l}\text { Mean } \pm \text { SD followup (mos) } \\
\text { UF: }\end{array}$ & \multicolumn{2}{|c|}{$15.2 \pm 17.2$} & \multicolumn{2}{|c|}{$36.7 \pm 30.2$} & 0.0001 \\
\hline $\begin{array}{l}\text { Mean } \pm \text { SD postop max } \\
\text { flow }(\mathrm{ml} / \mathrm{sec})\end{array}$ & \multicolumn{2}{|c|}{$26.2 \pm 12.3$} & \multicolumn{2}{|c|}{$11.9 \pm 6.9$} & 0.0001 \\
\hline Mean \pm SD postop VV $(\mathrm{ml})$ & \multicolumn{2}{|c|}{$356.3 \pm 180.2$} & \multicolumn{2}{|c|}{$297.0 \pm 139$} & 0.017 \\
\hline No. urinary symptoms (\%) & 25 & (12) & 54 & (86) & $<0.0001$ \\
\hline $\begin{array}{l}\text { No. obstructed voiding } \\
\text { curve }(\%)\end{array}$ & 27 & (13) & 58 & (92) & $<0.0001$ \\
\hline
\end{tabular}


Table 3

\section{UF vs RUG/VCUG findings}

\begin{tabular}{|c|c|c|c|c|}
\hline & $\begin{array}{c}\% \\
\text { Sensitivity }\end{array}$ & $\begin{array}{c}\% \\
\text { Specificity }\end{array}$ & $\begin{array}{c}\% \\
\text { PPV }\end{array}$ & $\begin{array}{c}\% \\
\text { NPV }\end{array}$ \\
\hline \multicolumn{5}{|l|}{ Flow rate $(\mathrm{ml} / \mathrm{sec})$ : } \\
\hline Less than 10 & 54 & 93 & 73 & 86 \\
\hline Less than 15 & 76 & 84 & 61 & 91 \\
\hline Less than 20 & 92 & 67 & 48 & 96 \\
\hline Obstructive voiding curve: & 93 & 84 & 71 & 97 \\
\hline + Flow rate less than $10 \mathrm{ml} / \mathrm{sec}$ & 54 & 94 & 76 & 86 \\
\hline $\begin{array}{l}\text { + Flow rate less than } 10 \mathrm{ml} / \mathrm{sec} \\
\text { + symptoms }\end{array}$ & 51 & 98 & 90 & 86 \\
\hline Symptoms: & 87 & 88 & 71 & 95 \\
\hline + Obstructive voiding curve & 76 & 96 & 87 & 92 \\
\hline \pm Obstructive voiding curve & 99 & 80 & 61 & 99 \\
\hline
\end{tabular}

